\title{
Editorial
}

\section{Open questions about influenza A (H1N1) 2009}

\author{
Miranda Robertson
}

When influenza A (H1N1) 2009 first started to spread on a pandemic scale earlier this year, we asked Peter Doherty and Stephen Turner to write a Question-and-Answer piece for us on the virus and the means to combat it [1]. Now, with the return to school of children at the start of the Northern Hemisphere autumn and the expected new wave of infections, we have published a follow-up Q\&A [2] on what we have learned about the virus since the last article, and the likelihood of an increase in the severity of the disease in the second wave of infections. For this, Doherty and Turner have recruited the help of colleagues Lorena Brown at Melbourne University and Anne Kelso at the WHO Collaborating Centre For Reference and Research on Influenza in Melbourne to deliver a quick overview of what is known, and their best guesses about the practical implications.

One question that they don't address however is whether the vaccines that have now been developed against influenza A (H1N1) 2009 will be available in time to prevent an epidemic. The vaccines are projected to be ready from early to mid-October, and it takes about two weeks for immunity to develop after vaccination, so if the wave of infections grows rapidly before the end of this month the vaccines will be too late to prevent widespread disease. Given the importance of formulating policies for distributing antiviral drugs and instituting social distancing (that is, in the first instance, closing schools), the pattern of viral spread and its consequences have been modelled under various assumptions about transmissibility, dependence of transmission on seasonality, proportion of the population immunized, and so on; and according to two modelling studies published in BMC Infectious Disease and BMC Medicine [3,4], and a report from the US President's Council of Advisors on Science and Technology (PCAST) [5], new infections may reach their peak in mid-October, just before vaccination campaigns can begin to take effect, and probably well before most people can be immunized. On the other hand, a model is only as reliable as the assumptions underlying it, and there is no certainty that the virus will beat the vaccines in what, inevitably, has been called a race against time.

By contrast it does seem certain that there will not be enough vaccine for everyone in the undeveloped world [6]. It is therefore very encouraging that preliminary results from two recent studies published in the New England Journal of Medicine suggest that one $15 \mu \mathrm{g}$ dose of inactivated viral vaccine, rather than the usual two [7], or two $7.5 \mu \mathrm{g}$ doses of vaccine with the adjuvant MF59 [8], may be sufficient to generate protective immunity. Since both studies measured the antibody response and not protection from infection (with which it is highly but not perfectly correlated), it is not yet certain that these vaccination protocols will actually be protective. But if they are, they will help stretch resources, not only of the vaccine, which is cumbersome to produce (see [1]), but also of funding and - if one dose will do - of time and organizational effort [9]. (Adjuvants are additives that stimulate more vigorous immune responses: the usual adjuvants for human vaccines are aluminium salts, whose mode of action is unknown [10]; but they do not work for influenza virus vaccines. MF59 is a more recently licensed oil-in-water emulsion that does work for influenza vaccines. For an earlier comment on what we don't know about adjuvants see [11].)

Not the least of the unanswered questions about pandemic influenza A (H1N1) 2009 is that of uptake. Now that people no longer routinely lose their schoolfriends or children to common childhood diseases that were endemic in the first half of the $20^{\text {th }}$ century, vaccination is too often seen as an imposition and a threat, partly because of publicity attendant on rare adverse reactions, or indeed upon conditions spuriously associated with vaccination. The recently reported death of a 14-year-old British girl some hours after vaccination against human papillomavirus (HPV) is therefore doubly unfortunate. The unexpected death of a young person is shocking at any time, but in most circumstances it is not likely to endanger other lives. It now seems clear that the death was due to an underlying health problem and the vaccination had nothing to do with it; but it is a fact of human psychology that the sudden death of a single teenage girl has more impact than a million still-living vaccinees perhaps ten of whom will be saved from early death from cervical cancer, and it is devoutly to be hoped that the arousal of public fears once again about the safety of vaccines will not jeopardize either the HPV or on the 'swine' 'flu vaccination programme.

Miranda Robertson, Editor

editorial@jbiol.com 


\section{References}

1. Doherty PC, Turner SJ: Q\&A: What do we know about influenza and what can we do about it? J Biol 2009, 8:46.

2. Turner SJ, Brown LE, Doherty PC, Kelso A: Q\&A: What have we found out about the influenza A (H1N1) 2009 pandemic virus? J Biol 2009, 8:69.

3. Flahault A, Vergu E, Boëlle PY: Potential for a global dynamic of influenza A (H1N1). BMC Infect Dis 2009, 9:129.

4. Balcan D, Hu H, Goncalves B, Bajardi P, Poletto C, Ramasco JJ, Paolotti D, Perra N, Tizzoni M, Van den Broeck W, Colizza $\mathrm{V}$, Vespignani A: Seasonal transmission potential and activity peaks of the new influenza A (H1N1): a Monte Carlo likelihood analysis based on human mobility. BMC Med 2009, 7:45.

5. Cohen $\mathrm{J}$ : A race against time to vaccinate against novel H1N1 virus. Science 2009, 325:1328-1329.

6. Yamada T: Poverty, wealth, and access to pandemic influenza vaccines. N Engl J Med 2009, 361:1129-1131.

7. Greenberg ME, Lai MH, Hartel GF, Wichems $\mathrm{CH}$, Gittleson $\mathrm{C}$, Bennet J, Dawson G, Hu W, Leggio C, Washington D, Basser $\mathrm{RL}$ : Response after one dose of a monovalent influenza $A$
(H1N1) 2009 vaccine - preliminary report. N Engl J Med 2009, 361:[Epub ahead of print].

8. Clark TW, Pareek M, Hoschler K, Dillon H, Nicholson KG, Groth N, Stephenson I: Trial of influenza A (H1N1) 2009 monovalent MF59-adjuvanted vaccine - preliminary report. $N$ Engl J Med 2009, 361:[Epub ahead of print].

9. Neuzil KM: Pandemic influenza vaccine policy Considering the early evidence. N Engl J Med 2009, 361:[Epub ahead of print].

10. McKee AS, Munks MW, Marrack P: How do adjuvants work? Important considerations for the new generation of adjuvants. Immunity 27:687-690.

11. Robertson M: Influenza: one or two more questions. $J$ Biol 2009, 8:45.

Published: 2 October 2009

doi:10.1186/jbiol182

(c) 2009 BioMed Central Ltd 